\title{
Efeito da Compatibilização da Mistura NBR/EVA sobre sua Morfologia de Fase Co-contínua
}

\author{
Bluma G. Soares \\ Instituto de Macromoléculas, UFRJ
}

Paulo J. de Oliveira

Departamento de Tecnologia Química, Universidade Federal Rural do Rio de Janeiro

\begin{abstract}
Resumo: O objetivo deste trabalho foi investigar a influência do poli (etileno-co-acetato de vinila) funcionalizado com grupos mercaptana (EVALSH) nas propriedades reológicas, morfológicas e dinamico-mecânicas de misturas envolvendo borracha nitrílica (NBR) e copolímero de etileno - acetato de vinila (EVA). Experiências envolvendo extração seletiva da fase NBR associadas à análise de microscopia eletrônica de varredura indicaram que estruturas do tipo co-contínuas são formadas em uma faixa de composição de 30 a 60 \% em massa de EVA. A adição de EVALSH não afetou esta faixa de co-continuidade, porém deslocou o limiar de percolação para menores teores de EVA. Este comportamento foi mais pronunciado em misturas ricas em NBR. A faixa de co-continuidade também foi evidenciada a partir de análise dinamico-mecânica. Dados reológicos revelaram também que a adição de EVALSH aumentou a viscosidade do sistema para misturas ricas em NBR.
\end{abstract}

Palavras-chave: NBR, EVA, compatibilização, co-continuidade, DMTA, reologia.

\section{Effect of the Compatibilization of NBR/EVA Blends on Co-continuous Morphology}

Abstract: The influence of ethylene-vinyl acetate copolymer functionalized with mercapto groups (EVALSH) on rheological, morphological and dynamic mechanical properties of nitrilic rubber (NBR)/ ethylene - vinyl acetate copolymer (EVA) blends has been investigated. Selective extraction experiments of NBR phase and scanning electron micrography suggest that co-continuous morphologies were formed in a range of blend compositions from 30 to $60 \mathrm{wt} \%$ of EVA. The addition of EVALSH did not affect the co-continuous range but shifted the percolation threshold of NBR-richer blends towards lower amount of EVA. The co-continuous range was also evidenced by dynamic mechanical analysis. Rheological data also revealed that the presence of EVALSH increased the viscosity of the blends containing higher amounts of NBR.

Keywords: NBR, EVA, compatibilization, co-continuity, DMTA, rheology.

\section{Introdução}

O desenvolvimento de novos materiais de engenharia a partir de misturas envolvendo dois ou mais polímeros, sem dúvida, constitui uma das mais importantes estratégias capazes de reunir, em um único material, propriedades específicas de cada um dos componentes, a custo reduzido. As propriedades mecânicas, térmicas e dinâmico-mecânicas dos mate- riais poliméricos estão intimamente correlacionadas com o tipo de morfologia presente na mistura ${ }^{[1]}$. Em geral, misturas poliméricas envolvendo um elastômero e um termoplástico são imiscíveis e podem apresentar morfologias do tipo fase dispersa ou co-contínua ${ }^{[2]}$. O tipo de morfologia formada é governado por vários fatores, tais como: condições de processamento, natureza dos polímeros, fração volumétrica de cada polímero e presença de agentes 
interfaciais ${ }^{[1-3]}$. Vários pesquisadores têm mostrado que a morfologia do tipo co-contínua pode ser obtida dentro de uma ampla faixa de composição ${ }^{[3-5]}$. Diversas hipóteses têm sido formuladas para tentar explicar a formação de estruturas co-contínuas. Uma delas baseia-se no estudo da inversão de fases, proposto por Paul e Barlow, em que os pesquisadores mostraram a existência de uma correlação entre o ponto de inversão de fase, a razão de viscosidade e a fração volumétrica de cada polímero na mistura ${ }^{[6]}$.

Para tentar compreender o início da formação das estruturas contínuas, Utracki direcionou seus estudos para investigação dos efeitos da coalescência a partir do aumento da concentração da fase dispersa ${ }^{[7]}$. Segundo Utracki, se a mistura é completamente imiscível, o início da formação de estruturas contínuas, também chamado limiar de percolação, pode ser compreendido analisando a geometria da partícula da fase dispersa que antecede à formação de uma estrutura contínua ${ }^{[7]}$. Para Utracki, a maioria das teorias desenvolvidas para definir o limiar de percolação deve incorporar o fenômeno de quebra e coalescência dos domínios. No entanto, os efeitos da coalescência são difíceis de serem explicados uma vez que dependem de vários fatores, tais como: o tempo, a característica da interface, a miscibilidade e a presença de agentes interfaciais ${ }^{[7]}$.

Nos últimos anos, vários trabalhos têm sido desenvolvidos na tentativa de aumentar a miscibilidade entre misturas envolvendo elastômeros e termoplásticos ${ }^{[8-9]}$. Este problema tem sido contornado com o uso de agentes compatibilizantes, os quais, quando adicionados à mistura, tendem a se localizar na interface, promovendo uma maior adesão entre as fases e conseqüentemente, uma maior estabilização da morfologia ${ }^{[8-10]}$. O efeito de reforço na interface é também denominado de compatibilização, uma vez que o aumento da interação entre as fases resulta em uma melhoria das propriedades da mistura ${ }^{[11]}$.

Vários pesquisadores têm mostrado que o estudo da formação de estruturas co-contínuas se torna mais complexo quando a interface em estudo sofre algum tipo de modificação, como por exemplo, a presença de agentes compatibilizantes ${ }^{[12,13]}$. Nestes casos, várias técnicas podem ser utilizadas em conjunto para tentar explicar os fenômenos de quebra e coalescência dos domínios, assim como a formação de estruturas co-contínuas. Dentre as técnicas utilizadas na avaliação de estruturas co-contínuas, destaca-se a técnica de extração seletiva ${ }^{[14]}$. Esta técnica é bastante útil, pois fornece informações sobre a morfologia no interior da mistura, e quando utilizada conjuntamente com os resultados de microscopia eletrônica de varredura (SEM) permite um estudo mais completo sobre a morfologia, uma vez que a técnica de SEM fornece somente uma avaliação da superfície da amostra.

Vários trabalhos apresentados na literatura mostram que a miscibilidade e a presença de agentes interfaciais produzem significativo efeito no comportamento reológico de misturas poliméricas ${ }^{[15-17]}$. Oommen e cols. investigaram o comportamento reológico da misturas NR/PMMA, na presença do agente interfacial NR-g-PMMA ${ }^{[17]}$. Os resultados obtidos neste estudo mostraram que a compatibilização produz um aumento na viscosidade da mistura ${ }^{[17]}$.

Nos últimos anos, a análise termodinâmico-mecânica (DMTA) tem sido amplamente utilizada no estudo da compatibilidade e tem permitido correlacionar as propriedades dinâmico-mecânicas dos materiais ao tipo de morfologia presente na mistura ${ }^{[18,19]}$.

Nosso grupo de pesquisa tem desenvolvido polímeros funcionalizados com grupos mercaptana para serem utilizados como agente interfacial em vários tipos de misturas poliméricas ${ }^{[20]}$. Recentemente, EVA hidrolisado e modificado com grupos mercaptana (EVALSH) foi utilizado na compatibilização da mistura $\mathrm{NBR} / \mathrm{EVA}^{[14]}$. O princípio da compatibilização baseia-se na possibilidade dos grupos $\mathrm{SH}$, presentes na cadeia de EVA, reagirem com a fase NBR, promovendo uma maior adesão entre as fases. Como resultado, observa-se uma melhora nas propriedades mecânicas dessas misturas.

Este trabalho tem como objetivo estudar o efeito do EVALSH na formação de estruturas co-contínuas em misturas envolvendo borracha nitrílica e poli(etileno-co-acetato de vinila) não vulcanizadas. Para atingir esse objetivo foram realizados ensaios utilizando-se as técnicas de extração seletiva, microscopia eletrônica de varredura, ensaios reológicos e dinâmico-mecânicos.

\section{Experimental}

\section{Material}

Borracha nitrílica (NBR), (NBR615) (33\% em peso de acrilonitrila; viscosidade Mooney $=32$ ) foi cedida pela Nitriflex Ind. Com. S.A. Copolímero de 
etileno - acetato de vinila (EVA18) (18\% em peso de acetato de vinila; índice de fluidez $=2,3 \mathrm{~g} / 10 \mathrm{~min}$ a $120^{\circ} \mathrm{C}$ ) foi cedido pela Petroquímica Triunfo S.A. EVA funcionalizado com grupos mercaptana (EVALSH) foi obtido em nosso laboratório, a partir da reação de esterificação de EVA hidrolisado e ácido mercaptoacético $^{[21]}$. EVASH usado neste estudo contém $62 \mathrm{mmol} \%$ de grupos mercaptana, determinado por análise termogravimétrica.

\section{Preparação das misturas}

As misturas foram preparadas em misturador de rolos do tipo Berstoff, a $110^{\circ} \mathrm{C}$ e $30 \mathrm{rpm}$. O processamento das misturas obedeceu ao seguinte procedimento: a NBR foi processada por 2 min e, em seguida, foram adicionados o EVALSH e o EVA, completando um tempo total de $5 \mathrm{~min}$ de processamento. Em seguida as amostras foram prensadas a $160^{\circ} \mathrm{C}$ durante $15 \mathrm{~min}$, a uma pressão de $15 \mathrm{MPa}$.

\section{Extração seletiva com solventes}

A inversão e a continuidade das fases foram determinadas submetendo as amostras à extração seletiva da fase NBR com metil-etilcetona (MEK), por uma semana, conforme descrito na literatura ${ }^{[14]}$. Após a extração, as amostras foram secas sob vácuo e pesadas. A quantidade de material não extraído é designada como fase contínua ${ }^{[6]}$. A presença de fases co-contínuas é caracterizada quando a fase NBR é totalmente extraída sem que ocorra a destruição da amostra. Nas composições em que o EVA está disperso na matriz de NBR, espera-se a total destruição da amostra, pois estando disperso, o EVA não é capaz de manter a estabilidade dimensional da amostra após a extração da matriz NBR. Por outro lado, quando a fase NBR encontra-se totalmente dispersa na matriz de EVA, o solvente seletivo para NBR não consegue penetrar e extrair a fase NBR que fica protegida pelo EVA. Neste caso, o peso da amostra antes e depois da extração é igual.

\section{Reologia}

Os ensaios de reometria capilar foram realizados em um reômetro capilar Instron (modelo 4204), segundo a norma ASTM D3835-93 ${ }^{[22]}$. A viscosidade das misturas NBR/EVA, previamente preparadas em misturador de rolos, foi avaliada a diferentes taxas de cisalhamento na temperatura de $150{ }^{\circ} \mathrm{C}$. Na realização desse ensaio, foi utilizado um capilar de razão
L/D igual a 100. As misturas foram pré-aquecidas por 3 min e, em seguida, submetidas ao cisalhamento. A força correspondente às diferentes velocidades de ensaios foi aferida e utilizada para determinação da tensão de cisalhamento aparente $\left(\tau_{\mathrm{w}}\right)$ e da taxa cisalhamento. Para eliminar o efeito de parede causado pelo capilar, determinou-se a taxa de cisalhamento corrigida, utilizando a equação estabelecida por Weissenberg-Rabinowish, Equação 1, onde $\gamma_{\mathrm{a}}$ é a taxa de cisalhamento aparente, $\gamma$ é a taxa de cisalhamento corrigida e $n$ é a tangente da curva obtida pela representação gráfica do logaritmo da tensão de cisalhamento em função da taxa de cisalhamento aparente. Após a correção da taxa de cisalhamento com o valor de $n$, a viscosidade das misturas foi obtida pela Equação 2[23].

$$
\gamma=\frac{(3 n+1)}{4 n} \times \gamma_{a}
$$

$$
\eta=\frac{\log (\tau)}{\log (\gamma)}
$$

\section{Análise dinâmico-mecânica}

As propriedades dinâmico-mecânicas das misturas foram medidas em um analisador dinâmico-mecânico Rheometric Scientific MKIII, na freqüência de $1 \mathrm{~Hz}$. As dimensões dos corpos de prova utilizados nestes ensaios foram de $5 \times 0,5 \times 0,05 \mathrm{~cm}^{3}$. Os ensaios foram realizados no modo "bending", na faixa de temperatura de $-70{ }^{\circ} \mathrm{C}$ a $50^{\circ} \mathrm{C}$, a uma taxa de aquecimento de $1^{\circ} \mathrm{C} / \mathrm{min}^{[14]}$.

\section{Estudo da Morfologia}

As morfologias das amostras foram observadas em um microscópio eletrônico de varredura, ZEISS DSM 960, utilizando-se a técnica de elétrons retroespalhados. As amostras prensadas foram submetidas a uma fratura criogênica, usando nitrogênio líquido. $\mathrm{O}$ contraste entre as fases foi obtido marcando-se a fase NBR com tetróxido de ósmio $\left(\mathrm{OsO}_{4}\right)$. Para isso, a superfície da fratura foi exposta à atmosfera de $\mathrm{OsO}_{4}$, por 4 horas. Após este tratamento, as amostras foram secas a vácuo e, em seguida, cobertas com carbono. Nestas micrografias, a fase clara corresponde à fase NBR, enquanto que a fase escura corresponde à fase EVA. 


\section{Resultados e Discussão}

\section{Estudos envolvendo extração seletiva}

A Figura 1 ilustra a variação da continuidade das fases EVA e NBR em função da composição da mistura. Quando a fase é rica em NBR, a continuidade da fase EVA começa a aumentar e atinge $100 \%$ de continuidade quando o teor de EVA atinge cerca de $30 \%$ em massa, tanto nas misturas compatibilizadas quanto nas misturas não compatibilizadas.

Para as misturas nas quais o teor de EVA é inferior a $30 \%$ em massa, verifica-se um comportamento diferenciado quando o EVALSH está presente na mistura. Tomando como exemplo a composição correspondente a $80 \%$ de NBR, observa-se que, na presença de EVALSH, a percentagem de fase contínua de EVA é igual a $51 \%$, enquanto que para mistura sem EVALSH este valor é de $20 \%$. Este resultado indica

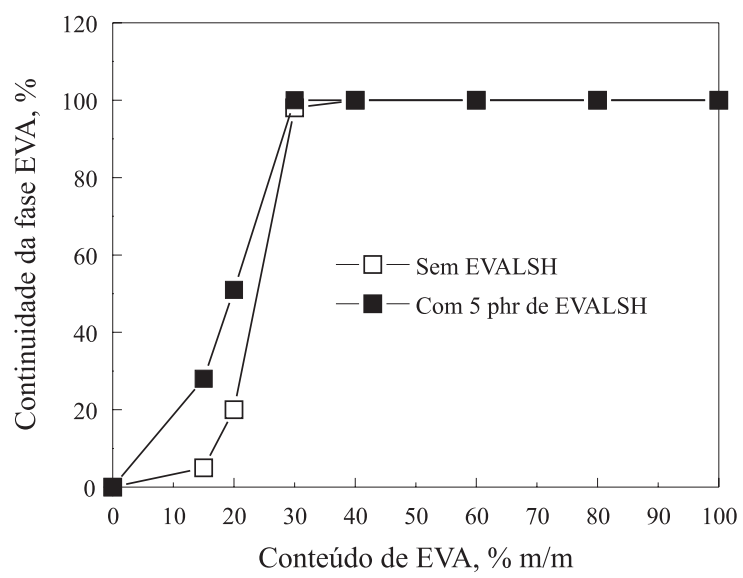

(a)

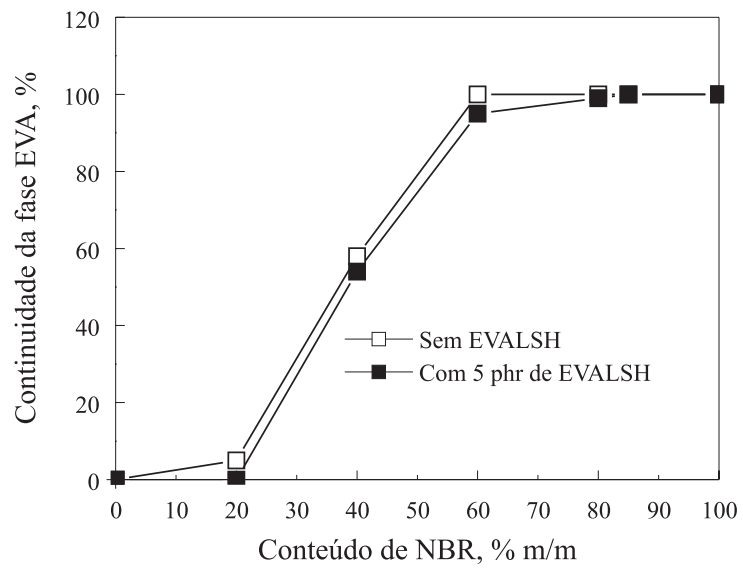

(b)

Figura 1. Variação da continuidade das fases EVA e NBR em função da composição da mistura. que o EVALSH promove um aumento na continuidade da fase EVA.

De acordo com Elmendorp e col., o desenvolvimento da morfologia em um sistema de mistura envolvendo dois polímeros está relacionado com o fenômeno de quebra dos domínios e coalescência da fase dispersa ${ }^{[24]}$. Segundo o pesquisador, a quebra dos domínios pode levar de vários segundos a várias horas. Quando este tempo excede ao da deformação dos domínios dispersos, observa-se a formação de domínios com estruturas contínuas ${ }^{[24]}$.

Com bases nesses argumentos, os resultados apresentados na Figura 1 sugerem que o produto formado pela reação entre o EVALSH e a matriz NBR aumenta a viscosidade da fase NBR, facilitando o escoamento e a coalescência da fase EVA. Além disso, acredita-se que o compatibilizante esteja também atuando no sentido de estabilizar a estrutura contínua de EVA.

A partir das experiências envolvendo extração seletiva, conclui-se que a faixa de co-continuidade estende-se de 70:30 até 40:60 NBR/EVA, e que a compatibilização não afeta esta faixa de co-continuidade. Para misturas ricas em EVA, a presença do agente compatibilizante não influencia o percentual de continuidade de cada fase.

Experimentos realizados por Thomas e cols. têm mostrado que a faixa de cocontinuidade encontra-se entre 40 e 60 em massa de $\mathrm{EVA}^{[25]}$. Nos estudos feitos por Thomas, as conclusões são baseadas nas análises feitas por microscopia eletrônica de varredura (SEM). Os resultados provenientes do uso de SEM baseiam-se em uma análise bidimensional da amostra, sem fornecer informações sobre a morfologia no interior da mesma. Desta maneira, informações mais



Figura 2. Co-continuidade da EVA em misturas NBR/EVA (80:20 \%) em função do conteúdo de EVALSH. 
precisas a respeito da morfologia na superfície e interior da amostra podem ser obtidas correlacionando os resultados obtidos por SEM e por extração seletiva.

$\mathrm{O}$ efeito do teor de EVALSH na formação de estruturas contínuas de EVA é ilustrado na Figura 2.

Nota-se que a continuidade da fase EVA aumenta com o teor de EVALSH na mistura, alcançando um máximo com a adição de 10 phr de EVALSH. Acima dessa concentração, ocorre uma diminuição da continuidade da fase EVA, provavelmente devido à perda da estabilidade morfológica, decorrente da saturação na interface. Esses resultados sustentam a hipótese de que, em misturas ricas em NBR, o EVALSH atua de forma promover uma maior estabilização da fase contínua de EVA.

\section{Estudos reológicos}

Vários pesquisadores têm mostrado que a adição de agentes compatibilizantes afeta o comportamento reológico das misturas ${ }^{[26-27]}$. A Figura 3 apresenta o comportamento da viscosidade da mistura NBR/EVA em função da composição, a duas taxas diferentes de cisalhamento. Observa-se que, para composições ricas em NBR, a presença de EVALSH promove um au-
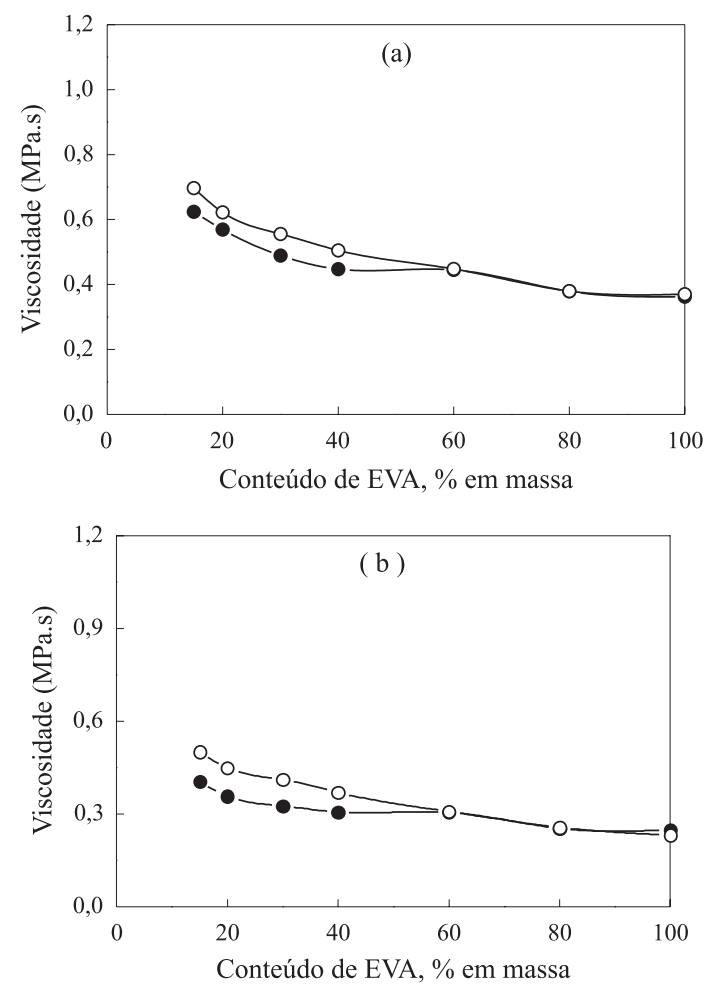

Figura 3. Variação da viscosidade da mistura NBR:EVA em função da composição, medidas a taxas de cisalhamento igual a: (a) $35,31 \mathrm{~s}^{-1} \mathrm{e}$ (b) $70,51 \mathrm{~s}^{-1}$

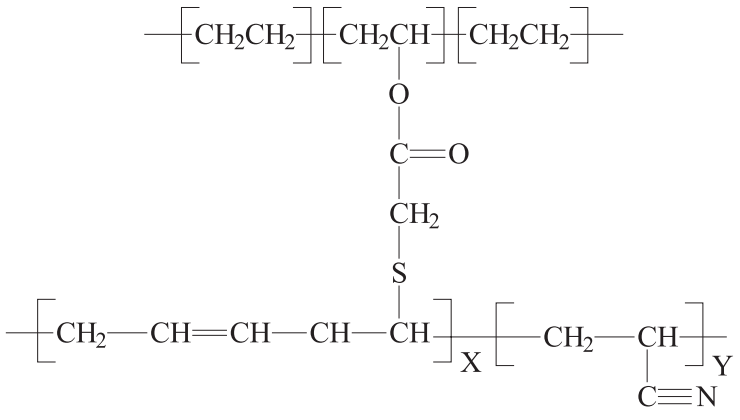

Figura 4. Reação entre a NBR e o EVALSH

mento na viscosidade da mistura. $\mathrm{O}$ aumento da viscosidade pode ser atribuído ao aumento no emaranhamento das cadeias poliméricas, como conseqüência do processo de compatibilização, promovido pelo EVALSH em decorrência das reações com a fase NBR, conforme sugerido na Figura 4. Entretanto, à medida que o teor de EVA na mistura aumenta, o efeito da compatibilização nesta propriedade torna-se cada vez menor.

Segundo a literatura, as reações químicas que ocorrem entre os componentes durante a compatibilização geralmente aumentam a viscosidade da mistura devido ao aumento da adesão interfacial, o que propicia uma efetiva transferência de energia entre a fase dispersa e a fase contínua ${ }^{[28,29]}$.

Os resultados de viscosidade associados àqueles obtidos por extração seletiva sugerem que o efeito do EVALSH é mais significativo para misturas ricas em NBR. Estes resultados concordam com aqueles relacionados às propriedades mecânicas e relatados em publicação anterior $^{[14]}$.

\section{Análise termodinâmico-mecânica}

As propriedades dinâmico-mecânicas dos materiais estão diretamente associadas à composição e ao tipo de interação presente na mistura e podem fornecer informações adicionais sobre a morfologia das misturas $^{[30]}$. A Figura 5 apresenta a dependência do fator de amortecimento ( $\tan \delta$ ) dos componentes puros (NBR e EVA) e das respectivas misturas não compatibilizadas, em função da temperatura. NBR apresenta um pico de $\tan \delta$ a cerca de $-16{ }^{\circ} \mathrm{C}$, relativa à transição vítrea do referido componente. Por outro lado, EVA apresenta duas transições a cerca de $-23{ }^{\circ} \mathrm{C}$ and $20^{\circ} \mathrm{C}$. As transições relacionadas ao EVA não são claramente perceptíveis, divido aos valores baixos de fator de amortecimento $(\tan \delta$ ) quando comparados àquele apresentado pelo NBR. 
As misturas apresentam um único pico, que pode ser atribuído principalmente à fase NBR. À medida que o teor de EVA na mistura aumenta, a temperatura correspondente ao máximo deste pico se desloca para regiões de menor temperatura. Este comportamento pode ser explicado com base nos coeficientes de expansão térmica da NBR e do EVA. Quando a amostra é resfriada abaixo das temperaturas de transição vítrea dos componentes, as fases se contraem. Este fenômeno pode ocasionar um aumento no volume livre da fase NBR, facilitando a mobilidade das cadeias correspondentes a essa fase. Este fenômeno é observado para composições ricas em NBR. Quando a proporção do componente EVA se situa na faixa de 30 a $60 \%$ em massa, o valor da Tg praticamente não se altera. Esta faixa de composição corresponde à estrutura co-contínua, conforme indicado nos ensaios de extração seletiva. Além disso, observa-se uma diminuição da intensidade da $\tan \delta_{\mathrm{NBR}}$ com o aumento da quantidade de EVA, devido ao decréscimo da fração volumétrica desta fase na mistura.

O efeito da estrutura co-contínua nas propriedades dinâmico-mecânicas dessas misturas é também ilustrado na Figura 6, onde o valor máximo da $\tan \delta_{\mathrm{NBR}}\left(\tan \delta_{\mathrm{MAX}}\right)$ é colocado em função da composição. É interessante notar que, na faixa de composição entre 70:30 e 60:40 de NBR/EVA, existe uma descontinuidade nos valores de $\tan \delta_{\mathrm{MAX}}$, em ambas as misturas, com e sem EVALSH.

De acordo com a literatura, a descontinuidade dos valores de $\tan \delta_{\mathrm{MAX}}$ com a composição está associada à formação de uma morfologia do tipo co-contínua ${ }^{[14]}$. Sendo assim, esses resultados estão de acordo com os resultados observados em nossos estudos sobre a

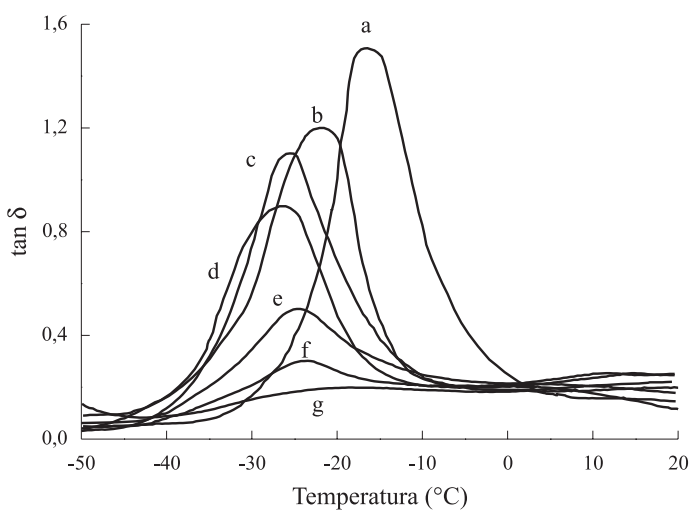

Figura 5. Variação da $\tan \delta$ com a temperatura para misturas de NBR/ EVA não compatibilizadas. (a) NBR puro, (g) EVA puro e misturas contendo: (b) $85 \%$, (c) $80 \%$, (d) $70 \%$, (e) $60 \%$ e (f) $40 \%$ em massa de NBR

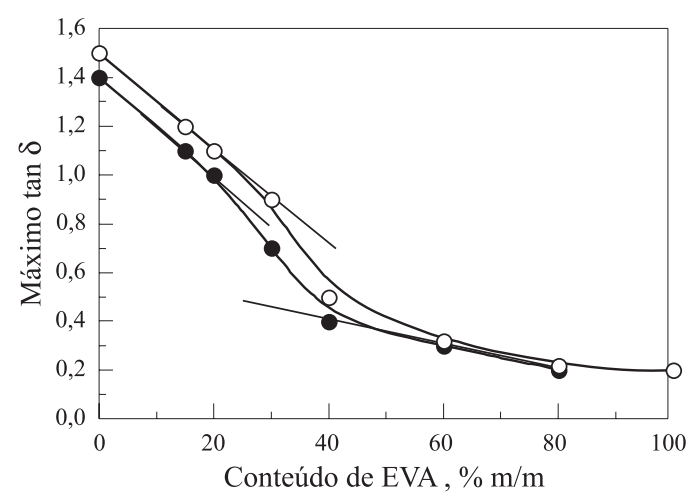

Figura 6. Variação do valor máximo da $\tan \delta_{\mathrm{NBR}}$, de acordo com a composição da mistura NBR/EVA. (O) sem EVALSH (•) com 5 phr de EVALSH

co-continuidade, utilizando a técnica de extração seletiva. Na Figura 6, observa-se também que a curva referente à mistura compatibilizada está deslocada para uma região rica em NBR, se comparada com a curva das misturas sem EVALSH. Esse resultado é semelhante ao observado em experiências com extração seletiva e confirma a ação do EVALSH como modificador da morfologia da mistura.

\section{Morfologia}

A Figura 7 mostra as morfologias das misturas NBR/EVA em função da composição e compatibilização, obtidas por microscopia eletrônica de varredura, utilizando a técnica de elétrons retroespalhados. A parte mais clara corresponde à fase NBR, tingida com tetróxido de ósmio e a parte mais escura é relacionada à fase EVA. As micrografias apresentadas no lado direito correspondem àquelas contendo $5 \mathrm{phr}$ de EVALSH. Na mistura NBR/EVA (80:20\%) (Figura 7a), a fase EVA encontra-se dispersa na fase NBR. A adição de 5 phr de EVALSH à mistura ocasiona a formação de pequenas estruturas contínuas de EVA na matriz NBR (Figura 7a'). Este resultado confirma as observações feitas no ensaio de extração seletiva, indicando que a presença de EVALSH promove uma maior estabilização da fase contínua de EVA. Quando o teor de EVA é igual a 40\% em massa, (Figura 7b), verifica-se a presença de uma morfologia co-contínua. A presença de EVALSH resulta em uma pequena coalescência da fase EVA (Figura 7b'). Nas Figuras $7 \mathrm{c}$ e c ${ }^{6}$, nota-se a ocorrência de inversão de fase. Esta composição apresenta, também, uma morfologia do tipo co-contínua. No entanto, na mistura com EVALSH, a fase NBR apresenta um tamanho menor e mais alongado, sugerindo que estas estruturas estão prestes a se romperem e formarem estruturas dispersas. 

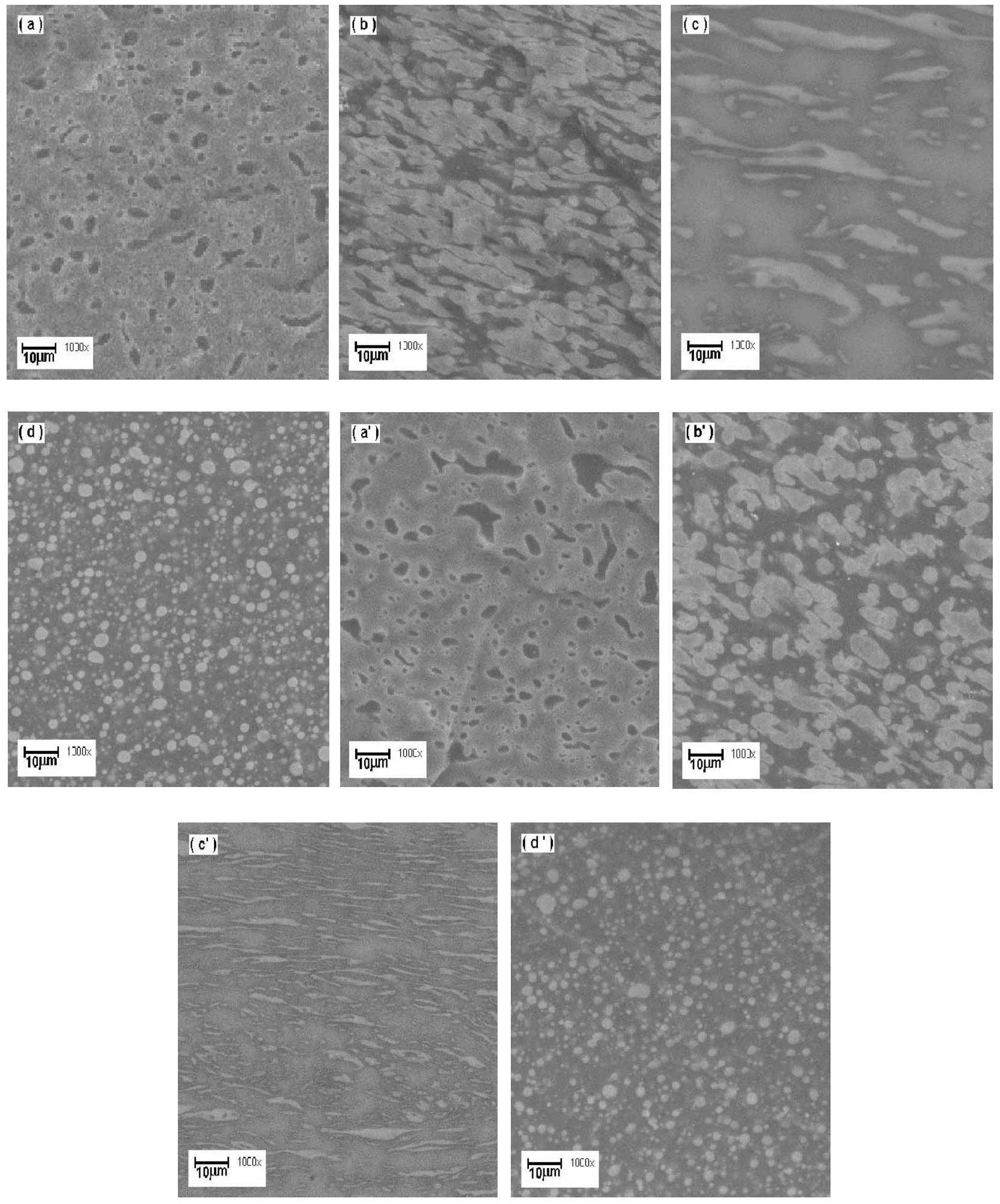

Figura 7. Microscopia eletrônica de varredura da misturas NBR/EVA (a) 80:20; (b) 60:40; (c) 40:60; (d) 20:80. (as letras a', b', c' e d' referem-se às misturas contendo $5 \mathrm{phr}$ de EVALSH).

Quando a fase NBR está dispersa na matriz EVA (Figuras $7 \mathrm{~d}$ e d`), a morfologia não é alterada significantemente pela adição do EVALSH. Esse resultado está de acordo com os obtidos nos ensaios de reometria capilar, em que nenhuma diferença entre os valores de viscosidade foi observada. 


\section{Conclusões}

Os resultados obtidos nesse estudo mostram que $o$ comportamento do fator de amortecimento $(\tan \delta)$ das misturas NBR/EVA é afetado pela co-continuidade das fases envolvidas na mistura. Com relação às experiências envolvendo extração seletiva sugere-se que a adição de EVALSH não altera a faixa de co-continuidade da mistura NBR/EVA, porém produz modificações no limite de percolação para misturas ricas em NBR. Neste caso, a fase EVA forma domínios mais alongados na presença de EVALSH, contribuindo para um aumento da continuidade desta fase. Este comportamento pode estar relacionado ao aumento da viscosidade da fase NBR, como indicado pelos resultados reológicos.

Para uma mesma composição de mistura, a adição de EVALSH diminuiu os valores de $\tan \delta_{\max }$ da fase NBR, indicando que o EVALSH atua no processo de relaxação da fase NBR, diminuindo a mobilidade desta fase. Este decréscimo na mobilidade pode estar relacionado à formação de estruturas reticuladas envolvendo NBR e EVALSH e contribui também para um acréscimo na viscosidade do sistema, como observado nos resultados de reometria capilar.

\section{Agradecimentos}

Os autores agradecem ao CNPq, CAPES, PADCT e CEPG-UFRJ, pelo apoio financeiro e à Nitriflex Ind. Com. SA e Petroquímica Triunfo pelas amostras de NBR e EVA, respectivamente.

\section{Referências Bibliográficas}

1. Jorgeseen, J. L.; Ransmunseen, K. L.; Chtcherbakova, E. A. \& Utracki, L. A. - Polym. Eng. Sci., 39, 1060 (1999)

2. Willemse, R. C.; Doer, A. P.; Van , J. D. \& Gostsis, A. D. Polymer , 40, 827 (1999).

3. Cor, K.; Martin, V. D.; Christopher, P. \& Robert, J. - Progress Polym. Sci., 23, 707 (1998)

4. Boutevin, B.; Piestrasanta, Y. \& Robin, J. J. -. Macromol. Symp., 57, 371 (1992).

5. Chung, O. \& Coran, A. Y. - Rubber Chem.Technol., 70, 781 (1997).

6. Paul, D. R. \& Barlow, J. W. - J. Macromol. Sci.: Macromol. Chem., C18, 109 (1980).

7. Utracki, L. A . - J. Rheol., 35, 1615 (1991).

8. Dedecker, K., Groeninckx, G. \& Inoue, T. - Polymer, 39, 5001 (1998).
9. Dedecker, K. \& Groeninckx, G. - Polymer 39,4993 (1998).

10. Jin, K. K.; Sanghyo, K.\& Park, C. E.- Polymer 38, 2155 (1998).

11. Xanthos, M. \& Dagli, S. S. - Polym. Eng. Sci., 31,932 (1991).

12. Asaletha, R.; Thomas, S.\& Kumaran, M.G.- Rubber Chem.Technol., 68, 671(1995).

13. Dedecker, K. \& Groeninckx, G.- Polymer 39, 4985 (1998).

14. Jansen, P. \& Soares, B. G. - J. Appl. Polym. Sci., 79,93 (2001).

15. Kim, Y. J.; Shim. G. S.; Lee,T. T. \& Kim, B. K. - J. Appl. Polym. Sci., 47, 295 (1993)

16. George, S.; Ramamurthy, K.; Anand, J. S.; Groeninckx, G.; Varghese, K. T. \& Thomas, S. - Polymer, 40, 4325 (1999).

17. Oommen, Z.; Premalatha, C.K.; Kuriakose, B. \& Thomas. S. - Polymer 38, 5611 (1997).

18. Wetton, R. E. \& Corish, P. J. - Polym. Testing, 8, 303 (1989).

19. Bandyopadhyay, G. G.; Bhagawan, S. S.; Ninan, K. N. \& Thomas, S. - J. Appl. Polym. Sci., 72, 165 (1999).

20. Oliveira M. G. \& Soares. B. G. - J. Appl. Polym.Sci, 82, 38 (2001).

21. Dutra, R.C.L., Lourenço, V.L., Diniz, M.F., Azevedo, M.F.P., Barbosa, R.V. \& Soares, B.G., Polym. Bull. 36, 593 (1996)

22. ASTM D3835-93a - "Standard Test Method for Determination of Properties of Polymeric Materials by Means of a Capillary Rheometer", American Society for Testing and Materials, section 9, Vol. 9.01, Philadelphia, ), (1986.)

23. Prentice, P.- "Rheology and its role in plastics processing", in: Rapra Review Reports, $\mathrm{N}^{\circ} 12,7$, .3 (1995).

24. Elmendorp, J. J.\& Malcke, R. J. - Polym Eng. Sci., 25, 1041(1985).

25. Varghese, H.; Bhagawan, S. S.; Rao, S. S. \& Thomas, S. Eur. Polym. Journal, 31, 957, (1995).

26. Koning, C.; Van, D. M.; Pagnoule, C. \& Jerome, R. - Polym. Eng. Sci., 30, 741 (1990).

27. Guriya, K. Ch.; Bhattachariya, A. K. \& Tripathy, D. K. Kautschuk Gummi Kunststoffe, 51, 134 (1998).

28. Miettinen, R. M. H.; Seppala, J.V.; Ikkala, O. T. \& Reima, I. T. - Polym. Eng. Sci., 34, 395 (1994).

29. George, S.; Neelakantan, N.R.; Varughese, K.T.; Thomas, S. - J. Polym. Sci. Part B: Polym. Physics 35, .2309 (1997)

30. Abhijit, J. H. A. \& Bhowmick, A. K -, Rubber Chem. Technol., 70, 798 (1997).

Recebido:03/05/02

Aprovado: 15/10/02 